- Holdren, director of the White House Office of Science and Technology Policy (OSTP). Noting the OSTP's defiance of a 2011 congressional rule that bans the office from collaborating with China (see Nature 478, 294-295; 2011), the House appropriations committee hacked away nearly one-third of the office's relatively small budget, leaving Holdren with $\$ 4.5$ million to run his agency.

Neal Lane, a senior fellow at the James A. Baker III Institute for Public Policy at Rice University in Houston, Texas, and a one-time science adviser to former US president Bill Clinton, calls this direct attack by the appropriations committee on a part of Obama's administration an unusual move that is "very counterproductive" for science policy, and will have damaging consequences for the OSTP and US-based research as whole. "It's the only place in the federal government where anybody is paying attention to the overall health of science and technology in the country," says Lane.

The action is one of many indications that the 2013 budget process, which will unfold during a presidential-election year, is likely to be hijacked and delayed by political manoeuvring. As Nature went to press, a bipartisan 'supercommittee' of legislators tasked with finding a way to reduce the federal deficit by $\$ 1.2$ trillion

\title{
A WORK IN PROGRESS
}

A spending bill enacted on 17 November includes 2012 budget allocations (US\$ millions) for several key federal science agencies. Still to come are final numbers for the National Institutes of Health, the Department of Energy and the Environmental Protection Agency, among others.

\begin{tabular}{|l|l|l|l|l|}
\hline Agency & $\mathbf{2 0 1 0}$ actual & $\begin{array}{l}\mathbf{2 0 1 1} \\
\text { estimated }\end{array}$ & $\begin{array}{l}\mathbf{2 0 1 2} \\
\text { requested }\end{array}$ & 2012 enacted \\
\hline NASA & 18,725 & 18,448 & 18,724 & 17,800 \\
\hline National Science Foundation & 6,873 & 6,860 & 7,768 & 7,033 \\
$\begin{array}{l}\text { National Oceanic and Atmospheric } \\
\text { Administration }\end{array}$ & 4,853 & 4,588 & 5,498 & 4,894 \\
$\begin{array}{l}\text { Food and Drug Administration } \\
\text { National Institute of Standards and } \\
\text { Technology }\end{array}$ & 2,362 & 2,447 & 2,744 & 2,497 \\
\hline
\end{tabular}

Sources: White House Office of Management and Budget; American Association for the Advancement of Science; FDA; US Senate Committee on Appropriations.

seemed all but certain to miss its 23 November deadline. This would trigger steep across-theboard cuts for all branches of the federal government, including all science-related agencies, beginning in January 2013 - unless Congress intervenes before then to overturn its own rules (see Nature 476, 133-134; 2011).

In the short term, legislators will move on to the next phase of their 2012 budget negotiations. By 16 December they must agree on a budget for those branches of the government not addressed in last week's spending bill. Among those are the Department of Energy and the Environmental Protection Agency, frequent victims of Republican attack that were marked for significant cuts in 2011. Also to be determined is the allocation for the National Institutes of Health, the federal government's largest research enterprise at $\$ 30$ billion. The agency is waiting to learn whether its ambitious bid to launch a centre for translational science will survive this year's budget battles (see Nature 477, 141-142; 2011).

Additional reporting by Susan Young and Meredith Wadman.

\section{Higgs hunt enters endgame}

\section{Large Hadron Collider could soon deliver a clear verdict on missing boson.}

\section{BY GEOFF BRUMFIEL IN GENEVA, SWITZERLAND}

$\mathrm{B}$ ill Murray is a man with secrets. Along with a handful of other scientists based at CERN, Europe's particle-physics facility near Geneva, Switzerland, Murray is one of the few researchers with access to the latest data on the Higgs boson - the most soughtafter particle in physics.

Looking at his laptop, he traces a thin black line that wiggles across a shaded area at the centre of a graph. This is the fruit of his summer's labours. "It's interesting, actually, looking at this again," he muses. A tantalizing pause. "But no, I can't say..."

Despite Murray's coyness, there are few places left for the Higgs to hide. Billed as the particle that helps to confer mass on other matter, and the final missing piece in the 'standard model' of particle physics, the Higgs would be a huge prize for CERN's Large Hadron Collider (LHC), the world's most powerful particle accelerator. But so far, the two massive detectors there ATLAS, where Murray works, and the Compact
Muon Solenoid (CMS) - have not seen any convincing signals of the elusive particle.

At a conference in Paris on 18 November, teams from ATLAS and the CMS experiments presented a combined analysis that wipes out a wide swathe of potential masses for the Higgs particle. Gone is the entire mass range from 141 to 476 gigaelectronvolts ( $\mathrm{GeV}$; energy and mass are interchangeable in particle physics). Together with earlier results from the 1990s, the analysis leaves a relatively narrow window of just $114-141 \mathrm{GeV}$ in which the Higgs could lurk (see 'Cornering the Higgs').

\section{CORNERING THE HIGGS}

Data from various particle accelerators restrict the mass of the Higgs particle, if it exists, to the range 114-141 gigaelectronvolts (GeV).

Large Electron-Positron Collider

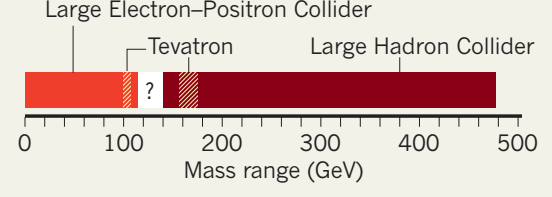

Analysis of the very latest data from this autumn - which Murray isn't yet ready to share - will scour the range that remains. If it turns out to be empty, physicists may have to accept that the particle simply isn't there. Working around the clock, the detector teams hope to have this larger data set analysed before the end of December. "We'll know the outcome within weeks," says Guido Tonelli, spokesman for the CMS detector.

\section{WAITING FOR GOD}

The quest for the Higgs boson, often called the 'God particle' after the title of a 1993 book by Nobel prizewinner Leon Lederman, is the public face of science at the LHC. Most highenergy physicists wince at the deistic designation, but they hold a near-religious devotion to the boson. Contrary to the popular view, their belief has less to do with mass than with fundamental forces.

Four fundamental forces are at work in nature: gravity, the strong nuclear force, the weak nuclear force and electromagnetism. Since the mid-1960s, physicists have strongly 


\section{DO YOU BELIEVE?}

Nature asked leading theoretical physicists whether they thought the Large Hadron Collider would find the Higgs particle predicted by the standard model of particle physics.
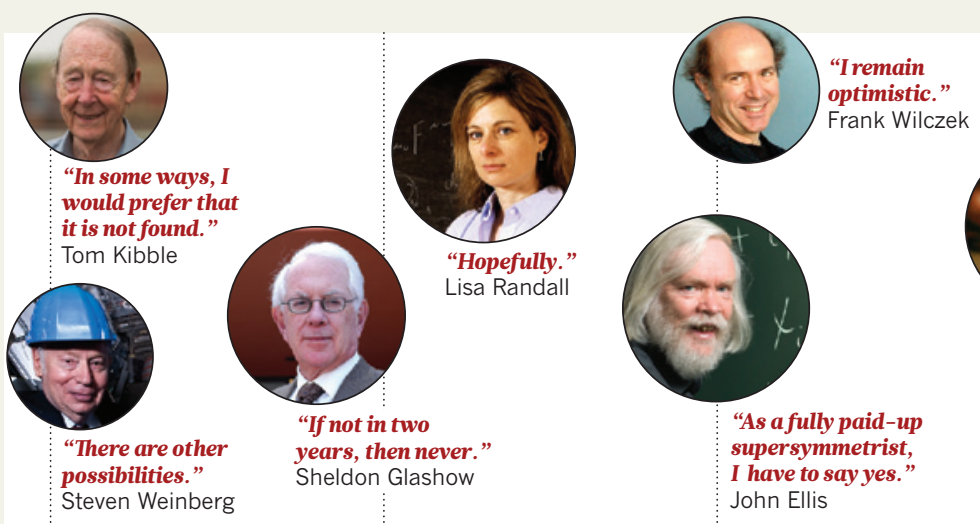

\section{$50 / 50$}

POTENTIALLY

YES

Tom Kibble, Imperial College London; Steven Weinberg University of Texas at Austin; Sheldon Glashow and Lisa Randall, Harvard University, Cambridge, Massachusetts; Frank Wilczek, Massachusetts Institute of Technology, Cambridge; John Ellis, CERN, Geneva, Switzerland; David Gross, Kavli Institute for Theoretical Physics, Santa Barbara, California.

suspected that the weak and electromagnetic forces are actually different aspects of a single 'electroweak' force. This is partly because the photon, the force-carrying particle of electromagnetism, is highly similar to the forcecarrying particles of the weak force - the W and $\mathrm{Z}$ bosons. Moreover, a single electroweak theory successfully predicts the interactions of fundamental particles.

There is one problem, however: the $\mathrm{W}$ and $\mathrm{Z}$ bosons are extremely heavy, nearly $100 \mathrm{GeV}$, whereas the photon is massless. To explain the difference, a number of physicists (including Peter Higgs in 1964) proposed a new field and particle. The eponymous Higgs mechanism would interact with the $\mathrm{W}$ and $\mathrm{Z}$ bosons, giving them mass, but would ignore the photon, allowing it to remain massless. Relatively straightforward tweaks to the Higgs machinery allow it to endow other particles, such as quarks, with their observed masses as well.

"The Higgs now sought at CERN is expected on the basis of the simplest picture" for the electroweak theory, says Steven Weinberg, a theorist who won a Nobel prize in 1979 for his work unifying electromagnetism and the weak force. "But there are other possibilities," he adds, reckoning the odds that the LHC's detectors will find the Higgs at 50/50 (see 'Do you believe?').

If there is no Higgs, then what? Gian Giudice, a theorist at CERN, recently published work suggesting that giant clusters of W bosons might serve the same purpose, but even he admits that "it would be a great surprise if it were true". Other models without the Higgs

\section{ONATURE.COM} For video interviews with Higgs hunters at CERN, visit: go.nature.com/ezpnaj boson invoke extra dimensions of space, but they are not yet sufficiently developed to guide experiments.

Perhaps the most likely alternative is that the Higgs is not a single particle, but rather a class of particles, which together do the job of unifying the two forces. Such a concept might appeal theoretically if a single Higgs is not found, but it would be a major headache for experimentalists to check. Theorists believe that the conventional Higgs boson would leave only a subtle mark on the detectors as it decays into $\mathrm{W}$ and $\mathrm{Z}$ bosons, high-energy photons and other particles. If there were two Higgs-like particles instead of one, the signal of each would be weaker still, says Murray. "It starts to get quite messy to do the analysis," he says.

The answer to the Higgs question lies in the data now being crunched at CERN and other academic-computing centres around the world. The first 70 trillion or so collisions turned up intriguing Higgs-like decays in the ATLAS and CMS experiments, hinting at a particle of around $140 \mathrm{GeV}$ (see Nature 475, 434; 2011). But the second batch of collisions showed nothing. If the collisions now being analysed show further evidence of Higgs decays, then the teams on the two experiments are likely to announce that they have found a tentative signal, to be firmed up in 2012. If not, the search will probably continue until the LHC is shut down for an upgrade at the end of next year.

Even if that continued search shows no evidence for a Higgs or anything else, the LHC will push on. Without a unified electroweak force, the standard model is unable to predict how certain particles and forces interact inside the collider, says Matthew Strassler, a theorist at Rutgers University in Piscataway, New Jersey. The LHC will gather data on exactly those processes, and that information could potentially be used to find a way in which electromagnetism and the weak force fit together. That process, Strassler adds, is likely to take many years. 